\title{
Next-generation, genome sequencing-based biomarkers: concerns and challenges for
}

\section{medical practice}

"Current efforts to complete the driver mutations catalogue for each common complex disease, and to understand biological and molecular networks provides an exciting field for novel biomarkers. However, the road to reaching medical applications is still long and many challenges must be overcome."

\section{KEYWORDS: biomarker $\approx$ genome sequencing $\approx$ genome-wide association studies}

In their day-to-day clinical practice, physicians are facing the adverse effects and limited effectiveness of currently used drugs in the treatment of major diseases. Biomarkers represent the great promise for improving prevention and treatment of complex common diseases including cancer, cardiovascular disease and diabetes. However, the clinical implications of basic and translational research efforts are modest. Given the genetic variation among patients with the same disease (phenotype) and the current genomic revolution that is enabling us to identify genetic differences, how optimistic can we be for a new generation of robust biomarkers in medicine?

The clinical need for prognostic and predictive markers has guided basic and translational sciences to offer clinicians new genetic and molecular tools that drive decision-making and allow tailoring of the best prevention or treatment in individual patients. The intensive research effort for developing biomarkers could be chronologically classified into four fields: the use of traditional clinicopathologic features and environmental factors, including lifestyle, classic single-gene molecular research, preliminary genome-wide association studies and complete genome sequencing and molecular networks, which are currently being used.

\section{Conventional clinical factors}

It is widely known that obesity, lack of physical activity, raised cholesterol, a low intake of fruit and vegetables, smoking, alcohol and several other factors increase the risk of common diseases such as cardiovascular diseases, diabetes and cancer. However, even using the combination of all these, along with conventional markers that use algorithmic approaches, is unable to accurately predict the disease risk in different individuals.

\section{Traditional single-gene molecular research}

The next research step focused on better understanding of molecular mechanisms underlying the pathogenesis of diseases. For example, prostate-specific antigen levels indicate a risk of prostate cancer and have been widely used for clinical screening. However, for complex multifactorial diseases, such as prostate cancer, the clinical use of such measurements has limitations for accurate individual risk predictions and general population screening [1]. In cancer treatment, the tumor node metastasis staging system and clinicopathologic features are the standard tools currently used for surgery, radiotherapy and systemic therapeutic decisions [2], but its modest ability to discriminate between responder and nonresponder patients is widely recognized.

Several research strategies and scientific fields have been developed for personalized medicine. Pharmacogenetics, studying genetic associations with drug efficacy and toxicity, has led to the development of several enzymes with the most important belonging to the family of cytochrome P450 [3]. The primary aim is to predict the adverse effects for the guidance of individualized treatment. Although the term pharmacogenomics overlaps with pharmacogenetics, it is distinct owing to the fact that it evaluates the application of genomics to drug discovery. Pharmacogenomics evaluates the mechanism of action of drugs on cells as revealed by geneexpression patterns. Pharmacoproteomics provides a more functional representation of patientto-patient variation than that provided by genotyping, which contributes to personalized medicine. A 'pharmacometabonomic' approach involves the study of metabolites and how these can contribute to personalizing drug treatment.

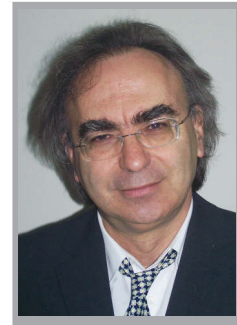

Dimitrios H Roukos Personalized Cancer Medicine, Biobank, loannina University School of Medicine, loannina, 45110 Greece and Department of Surgery, loannina University School of Medicine, loannina, 45110 Greece Tel.: +302651007423 Fax: +30 2651007094 droukos@uoi.gr 


\section{Multigene assays \& genome-wide association studies}

The advent of high-throughput technology over the past 10 years has revolutionized basic and translational research strategies. The massively parallel sequencing platforms allow the profiling of the expression of hundreds of genes at a time. Several gene-expression profiling studies using microarrays and real-time PCR techniques have resulted in the development of a molecular classification of breast cancer and multigene assays. It was believed that such a multigene approach could lead to more individualized therapeutic decisions in an era of personalized medicine.

Of the multiple multigene assays proposed to have prognostic or predictive value for many solid tumors, including breast cancer, two gene signatures have most rapidly progressed: the 21-gene assay and the 70-gene signature. The 21-gene recurrence score was developed to identify high-risk patients of distant recurrence among those with node-negative, estrogen receptor-positive early breast cancer [4]. The efficacy of this genetic tool, which has now been commercialized (Oncotype DX), is tested in the large-scale Phase III trial (Trial Assigning Individual Option for Treatment [TAILORx]) in the USA. The 70-gene signature has been approved by the US FDA as a genetic test for women with node-negative early breast cancer. It was believed that it would be a robust predictor of distant metastatic risk, enabling adjuvant chemotherapy for high-risk patients [5]. This genetic tool has been commercialized (MammaPrint) and is now being tested in a Phase III randomized controlled trial in Europe (Microarray in Node-Negative Disease Avoids Chemotherapy [MINDACT] trial).

However, gene-expression profiling studies alone have substantial limitations. There are two main arguments for the uncertainty of the clinical utility of these multigene classifiers. There is a series of methodological disadvantages in both the original training sets developed and the subsequent observational validation studies to assess the predictive power of these classifiers [6,7]. However, the clinical utility of Oncotype Dx and MammaPrint assays is not known and only the result of the ongoing Phase III trials will definitely indicate the robustness of these genetic tools.

In the prevention setting, genome-wide association studies (GWAS) with potential identification of genetic variation for disease risk prediction over the last 3 years have been met with enthusiasm. Based on sequence databases, microchips with tens of thousands of genetic variants, including single-nucleotide polymorphisms and copy-number variants were developed. These chips have allowed GWAS to identify a wealth of novel genetic variants associated with diseases. As a result, biotechnology companies, including Celera (CA, USA) and deCODE Genetics (Reykjavik, Iceland), have had to rethink their optimistic assumption that selling human genetic information could turn a huge profit from personal genetic testing.

Summarizing the results of a GWAS examining personalized risk prediction, two categories of genetic variants have emerged. The first group includes very rare mutations in the general population that raise the risk of diseases, such as schizophrenia, epilepsy and autism, by up to 20 -fold. These discoveries have clinical applications in risk prediction, but owing to their rarity, the benefits are limited to only a few individuals. The second includes common genetic variants, but they confer only a very small increase in disease risk and, therefore, have little or no clinical utility $[8,9]$. Indeed, recently, nine and ten single-nucleotide polymorphisms identified by GWAS to be involved in cardiovascular disease and breast cancer, respectively, did not improve disease risk predictions in two large studies $[10,11]$.

\section{Complete genome sequencing}

When the first draft of the complete human genome sequence was announced, The White House press statement articulated the hope, felt by many, that this landmark achievement would "lead to a new era of molecular medicine, an era that will bring new ways to prevent, diagnose, treat and cure disease". Today, 10 years on, where do we stand and where we will be 10 years from now?

\section{Sequencing technology explosion}

The first postgenome decade was characterized by spectacular advances in genome science. The dramatic improvement in DNA-sequencing technology along with a dramatic drop of sequencing costs has increased the numbers of base pairs sequenced in databases from 8 billion in 2000 to 270 billion today [12]. To date, there have been two dozen publications of complete-sequence human genomes and close to 200 unpublished ones [13]. Several other international projects and consortia, such as the Encyclopedia of DNA Elements (ENCODE), provide important information on coding and noncoding DNA, aiming to improve our understanding of every functional element in the 
human genome. Much noncoding DNA may have a regulatory role; small RNAs of different varieties appear to control gene expression at the level of both DNA and RNA transcription in ways that are still only beginning to become clear [14].

As complete-genome sequencing data emerge, it becomes clear that the more we learn, the bigger the problem of understanding the life mysteries of biodiversity, aging and complex disease pathogenesis and evolution, such as cancer, becomes [15]. However, the impact of the genomic revolution on medicine and health is limited. To move forward to the future, Collins considers five important key lessons: personalized medicine, technology, policy, partnerships and pharmacogenomics [16]. Venter emphasizes the need for research in linking genotype to phenotype and points out that owing to the myriad of phenotypic traits, more powerful computational strategies will be needed to link phenotype to genotype [17].

\section{Predicting the genotype-phenotype relationship}

Indeed, there are several reasons explaining the limitations in translating genomic discoveries into medical practice. Major hurdles that must be overcome in order to develop robust biomarkers for improving health in the future can be summarized into two directions. First, to complete the somatic mutation catalogue of the disorders. Second, to understand complex interactions between these genetic variants, between genes and environment or lifestyle, and between various human cells.

\section{Challenges}

Many challenges exist despite advances with the latest technology. First, DNA changes include not only point mutations, such as nucleotide insertions, deletions and single-nucleotide polymorphisms, but also genomic rearrangements and copy-number changes. For example, complete genome sequencing for breast, lung and other major cancers have revealed a huge number of mutation and widespread variability [18-24]. Second, the discrimination between causal (driver) mutations and non-causal (passenger) mutations that are identified by genome sequencing studies is still difficult. Third, the bigger challenge is to understand how the complex gene-gene, protein-protein, gene-environment and intratumoral cell-cell interactions occur in a timely and very dynamic process, for example in cancer.

\section{Molecular networks}

Not only genotyping but also phenotype data are crucial for disease risk prediction and progression in individual persons $[17,25]$. As the costs drop and the quality of sequencing data are improved, the catalogue of driver mutations for major diseases will be improved. High-quality clinical and therapeutic data (phenotype) are available from large-scale randomized controlled trials and databases. The bigger challenge is how to link all these data with genotyping.

However, the nonlinear relationship between genotype and phenotype represents the biggest challenge in biomedical and mathematical sciences. Several computational strategies are being developed to predict genegene and gene-environment interactions [26]. Bionetwork modeling represents one of the most promising research fields towards genotype-phenotype-based personalized medicine [27]. Efforts are underway to integrate genotyping and molecular data into the modeling of molecular networks to predict outcomes [27]. Systems biology, oncology and medicine open new ways to understand complex biological systems and explain how it could be possible to link genomic data with clinical data and diseases for understanding pathogenesis and developing biomarkers for disease risk prediction and prevention [9,26-37].

\section{Conclusion}

Physicians urgently need biomarkers for improving the healthcare of their patients. However, despite effort with traditional single-gene molecular research and preliminary GWAS, clinical implications are modest and valid concerns exist for this research direction.

Current efforts to complete the driver mutations catalogue for each common complex disease, and to understand biological and molecular networks provides an exciting field for novel biomarkers. However, the road to reaching medical applications is still long and many challenges must be overcome.

Financial \& competing interests disclosure

The author has no relevant affiliations or financial involvement with any organization or entity with a financial interest in or financial conflict with the subject matter or materials discussed in the manuscript. This includes employment, consultancies, honoraria, stock ownership or options, expert testimony, grants or patents received or pending, or royalties.

No writing assistance was utilized in the production of this manuscript. 


\section{Bibliography}

1 Lin K, Lipsitz R, Miller T, Janakiraman S; US Preventive Services Task Force: Benefits and harms of prostate-specific antigen screening for prostate cancer: an evidence update for the U.S. Preventive Services Task Force. Ann. Intern. Med. 149(3), 192-199 (2008).

2 Edge SE, Byrd DR, Carducci MA, Compton CA (Eds): AJCC Cancer Staging Manual (7th Edition). Springer, NY, USA (2009).

3 Roederer MW: Cytochrome P450 enzymes and genotype-guided drug therapy. Curr. Opin. Mol. Ther. 11(6), 632-640 (2009).

4 Paik S, Shak S, Tang G et al.: A multigene assay to predict recurrence of tamoxifentreated, node-negative breast cancer. $N$. Engl. J. Med. 351, 2817-2826 (2004).

5 van de Vijver MJ, He YD, van't Veer LJ et al.: A gene-expression signature as a predictor of survival in breast cancer. N. Engl. J. Med. 347, 1999-2009 (2002).

6 O’Shaughnessy JA: Molecular signatures predict outcomes of breast cancer. $N$. Engl. J. Med. 355(6), 615-617 (2006).

7 Huang CC, Bredel M: Use of gene signature to improve risk estimation in cancer. JAMA 299(13), 1605-1606 (2008).

8 Goldstein DB: 2020 visions. Personalized medicine. Nature 463(7277), 26-32 (2010).

9 Roukos DH: Genome-wide association studies: how predictable is a person's cancer risk? Expert Rev. Anticancer Ther. 9(4), 389-392 (2009).

10 Kathiresan S, Melander O, Anevski D et al: Polymorphisms associated with cholesterol and risk of cardiovascular events. $N$. Engl. J. Med. 358(12), 1240-1249 (2008).

11 Wacholder S, Hartge P, Prentice R et al.: Performance of common genetic variants in breast-cancer risk models. N. Engl. J. Med. 362(11), 986-993 (2010).

12 No authors listed. Human genome at ten: the sequence explosion. Nature 464(7289), 670-671 (2010).

13 No authors listed. The human genome at ten. Nature 464(7289), 649-650 (2010).
14 Check Hayden E: Human genome at ten: life is complicated. Nature 464(7289), 664-667 (2010).

15 Ledford H: Big science: the cancer genome challenge. Nature 464(7291), 972-974 (2010).

16 Collins F: Has the revolution arrived? Nature 464(7289), 674-675 (2010).

17 Venter JC: Multiple personal genomes await. Nature 464(7289), 676-677 (2010).

18 Stratton MR, Campbell PJ, Futreal PA: The cancer genome. Nature 458(7239), 719-724 (2009).

19 Pleasance ED, Stephens PJ, O'Meara S et al.: A small-cell lung cancer genome with complex signatures of tobacco exposure. Nature 463(7278), 184-190 (2010).

20 Pleasance ED, Cheetham RK, Stephens PJ et al.: A comprehensive catalogue of somatic mutations from a human cancer genome. Nature 463(7278), 191-196.

21 Stephens PJ, McBride DJ, Lin ML et al: Complex landscapes of somatic rearrangement in human breast cancer genomes. Nature 462 (7276), 1005-1010 (2009).

22 Beroukhim R, Mermel CH, Porter D et al.: The landscape of somatic copy-number alteration across human cancers. Nature 463(7283), 899-905 (2010).

23 Ding L, Ellis MJ, Li S et al:: Genome remodelling in a basal-like breast cancer metastasis and xenograft. Nature 464(7 291), 999-1005 (2010).

24 Jones S, Zhang X, Parsons DW: Core signaling pathways in human pancreatic cancers revealed by global genomic analyses. Science 321(5897), 1801-1806 (2008).

25 Roukos DH: Novel clinico-genome network modeling for revolutionizing genotypephenotype-based personalized cancer care. Expert Rev. Mol. Diagn. 10(1), 33-48 (2010).

26 Rockman MV: Reverse engineering the genotype-phenotype map with natural genetic variation. Nature 456, 738-744 (2008).
27 Roukos DH: Bionetworks-based personalized medicine versus comparative-effectiveness research or harmonization of both in cancer management? Expert Rev. Mol. Diagn. 10(3), 247-250 (2010).

28 Roukos DH: Systems medicine: a real approach for future personalized oncology? Pharmacogenomics 11(3), 283-287 (2010).

29 Schadt EE: Molecular networks as sensors and drivers of common human diseases. Nature 461(7261), 218-223 (2009).

30 Weinberg R: Point: hypotheses first. Nature 464(7289), 678 (2010).

31 Roukos DH, Ziogas D: From tumor size and HER2 status to systems oncology for very early breast cancer treatment. Expert Rev. Anticancer Ther. 10(2), 123-128 (2010).

32 Roukos DH: Breast cancer outcomes: the crucial role of the breast surgeon in the era of personal genetics and systems biology. Ann. Surg. 249(6), 1067-1068 (2009).

33 Ziogas D, Roukos DH: Genetics and personal genomics for personalized breast cancer surgery: progress and challenges in research and clinical practice. Ann. Surg. Oncol. 16(7), 1771-1782 (2009).

34 Roukos DH: Personalized cancer diagnostics and therapeutics. Expert Rev. Mol. Diagn. 9(3), 227-229 (2009).

35 Roukos DH: Beyond HER2 and trastuzumab: heterogeneity, systems biology, and cancer origin research may guide the future for personalized treatment of very early but aggressive breast cancer. J. Clin. Oncol. 28(17), E279-E280 (2010).

36 Roukos DH: Targeting gastric cancer with trastuzumab: new clinical practice and innovative developments to overcome resistance. Ann. Surg. Oncol. 17, 14-17 (2010).

37 Roukos DH: Personal genomics and genome-wide association studies: novel discoveries but limitations for practical personalized medicine. Ann. Surg. Oncol. 16(3), 772-773 (2009). 